\title{
Controlling the Magnetization Direction in Molecules via Their Oxidation State
}

\author{
N. Atodiresei, ${ }^{1, *}$ P. H. Dederichs, ${ }^{1}$ Y. Mokrousov, ${ }^{2}$ L. Bergqvist, ${ }^{1}$ G. Bihlmayer, ${ }^{1}$ and S. Blügel ${ }^{1}$ \\ ${ }^{1}$ Institut für Festkörperforschung, Forschungszentrum Jülich, D-52425 Jülich, Germany \\ ${ }^{2}$ Institute for Applied Physics, University of Hamburg, 20355 Hamburg, Germany
}

(Received 17 November 2007; published 21 March 2008)

\begin{abstract}
By means of $a b$ initio calculations we predict that it is possible to manipulate the magnetization direction in organic magnetic molecules by changing their oxidation state. We demonstrate this novel effect on the $\mathrm{Eu}_{2}\left(\mathrm{C}_{8} \mathrm{H}_{8}\right)_{3}$ molecule, in which the hybridization of the outer $\pi$ ring states with the Eu $4 f$ states causes a redistribution of the orbitals around the Fermi level leading to a strong ferromagnetism due to a hole-mediated exchange mechanism. As a key result, we predict an oscillatory behavior of the easy axis of the magnetization as a function of the oxidation state of the molecule - a new effect, which could lead to new technological applications.
\end{abstract}

PACS numbers: 75.75.+a, 75.50.Xx, 85.35.Gv, 85.65.+h

The miniaturization of the structures used in modern ultra-high-density magnetic recording aims at nonvolatility, low-energy consumption, and short access times for reading and writing. The nanostructured layered inorganic magnetic materials will reach soon the limit of nanopatterning [1], which motivates the search for completely new approaches. In this respect, organic molecular magnets are very promising candidates as working units for spintronics [2-4]. From the technological functionality point of view, besides a small size, they are required to have a strong ferromagnetic coupling of local spin moments and large values of magnetic anisotropy energy (MAE), a quantity crucial for practical applications. MAE gives an estimate for the energy barrier necessary to stabilize the magnetic moments against quantum tunneling and thermal fluctuations.

However, an easy way to control the spin polarized electrons in downscaling devices has proved to be quite a challenge. In particular, the effective ways of dynamical control of the magnetization direction in small magnetic bits used for storage and transport applications are still to be established. Because of the reduced dimensions, for the high-temperature applications the magnetic bits have to exhibit huge values of magnetic anisotropy to resist the temperature fluctuations. Moreover, controlling the magnetization direction, electronic and transport properties of the bits have to be easily manageable and should avoid applying enormous external magnetic fields due to huge anisotropies [5]. At the moment, temporary solutions of this problem lie in the heat-assisted magnetic recording [6] and in the writing assisted by microwave excitation [7]. But even in such promising techniques the presence of the magnetic field is necessary.

In this Letter we propose a simple way to control the magnetization direction in organic magnetic molecules via a basic mechanism, which is common for the biomolecular world: an oxidation-reduction reaction. The transfer of electrons in or out of the molecule, the heart of an oxidation-reduction process, normally modifies not only the electronic properties of the molecule, but can also influence its magnetism drastically. Here we demonstrate that for a special class of ferromagnetic organic molecules the transfer of electrons in or out of the molecule results in a drastic change in the magnetization direction, preserving the large spin due to a strong ferromagnetic coupling and sizable magnetic anisotropies.

To prove these effects, we performed first principles studies on the $\mathrm{Eu}_{2}\left(\mathrm{C}_{8} \mathrm{H}_{8}\right)_{3}$ molecule [8-10], its corresponding cations $\left[\mathrm{Eu}_{2}\left(\mathrm{C}_{8} \mathrm{H}_{8}\right)_{3}^{+1}, \mathrm{Eu}_{2}\left(\mathrm{C}_{8} \mathrm{H}_{8}\right)_{3}^{+2}\right]$ and anions $\left[\mathrm{Eu}_{2}\left(\mathrm{C}_{8} \mathrm{H}_{8}\right)_{3}^{-1}, \mathrm{Eu}_{2}\left(\mathrm{C}_{8} \mathrm{H}_{8}\right)_{3}^{-2}\right]$. The $\mathrm{Eu}_{2}\left(\mathrm{C}_{8} \mathrm{H}_{8}\right)_{3}$ molecule contains $\mathrm{Eu}$ as rare earth atom and has single spin characteristics with a large spin quantum number, much larger than those of known conventional ferromagnetic materials. As we will demonstrate in this Letter the $\mathrm{Eu}_{2}\left(\mathrm{C}_{8} \mathrm{H}_{8}\right)_{3}$ molecule exhibits an extremely interesting phenomenon: the magnetization orientation depends on the oxidation state of the molecule $(+2,+1,0$, or -1$)$ revealing an oscillating behavior from in the plane to out of plane and it is stabilized by large values of the magnetic anisotropy.

$A b$ initio calculations were performed within the framework of density functional theory [11] in the generalized gradient approximation [12]. The Kohn-Sham equations have been solved self-consistently using the projector augmented plane-wave method (PAW) [13] as implemented in the VASP code [14]. In order to take into account the proper orbital dependence of the Coulomb and exchange interaction we employed the LDA $+U$ method in treating the $\mathrm{Eu}$ $4 f$ states [15], which improves not only the description of the ground state properties such as magnetic moments and interatomic exchange parameters, but also excited states and energy gaps $[15,16]$. The photoelectron spectra for the $\mathrm{Eu}_{n}\left(\mathrm{C}_{8} \mathrm{H}_{8}\right)_{n+1}^{-1}(n=1-7)$ sandwiches show a photodetachment contribution of $4 f$ orbitals between $3.0-4.0 \mathrm{eV}$ $[8,10]$. More precisely, a value of $3.7 \mathrm{eV}$ corresponds to the photodetachment energy in one-end double sandwich anion $\mathrm{Eu}_{2}\left(\mathrm{C}_{8} \mathrm{H}_{8}\right)_{2}^{1-}$ [10]. Here we present the results with the $U=3.7 \mathrm{eV}$, but we have carefully checked the consistency of our results for a set of $U$ values between 
2.7 and $10.7 \mathrm{eV}$. We considered two possible symmetrydetermined directions of the magnetization in the molecules: axial $(z)$-perpendicular to the plane of the $\mathrm{C}_{8} \mathrm{H}_{8}$ ring and radial $(r)$-in the plane of the $\mathrm{C}_{8} \mathrm{H}_{8}$ ring. The MAE is defined as the total energy difference between these two configurations. The magnetic dipolar contribution to the MAE is negligible; therefore, only the spin-orbit coupling is responsible for the anisotropy barrier [17]. The structures were relaxed including spin-orbit coupling without any symmetry restrictions.

Our calculations confirm that the structures of the $\mathrm{Eu}_{2}\left(\mathrm{C}_{8} \mathrm{H}_{8}\right)_{3}$ molecule and its ionic species are sandwiches with the metal atom above the center of gravity of $\mathrm{C}_{8} \mathrm{H}_{8}$ ligand molecules [8-10]. All the $\mathrm{Eu}_{2}\left(\mathrm{C}_{8} \mathrm{H}_{8}\right)_{3}$ ionic species have a ferromagnetic ground state, with the total spin moment increasing linearly with the number of additional electrons: from $10 \mu_{B}$ for $\mathrm{Eu}_{2}\left(\mathrm{C}_{8} \mathrm{H}_{8}\right)_{3}^{+2}$ cation up to $14 \mu_{B}$ for the $\mathrm{Eu}_{2}\left(\mathrm{C}_{8} \mathrm{H}_{8}\right)_{3}^{-2}$ anion. The total energy difference between the ferromagnetic (FM) and antiferromagnetic (AFM) spin configuration lies in the range of 100$120 \mathrm{meV}$ for all ionic species, with the exception of the $\mathrm{Eu}_{2}\left(\mathrm{C}_{8} \mathrm{H}_{8}\right)_{3}^{-2}$ anion for which the energy difference between FM and AFM magnetic states vanishes.

The charge-difference plots of the molecule against the sum of the separated systems, $\mathrm{Eu}_{2}$ and $\left(\mathrm{C}_{8} \mathrm{H}_{8}\right)_{3}$, together with the analysis of the charge transfer in or out of the pseudopotential spheres confirm a strong ionic character of the bonding between $\mathrm{Eu}$ and $\mathrm{C}_{8} \mathrm{H}_{8}$ ligands $[8-10,18]$. The $\mathrm{Eu} 6 s$ electrons are transferred to an aromatic type molecular orbital characterized by a high density of states of the $\mathrm{C}$ sites; therefore, the Eu atom can formally be seen as a +2 positive ion [10].

The ferromagnetic local density of states and plots of the molecular orbitals (see Fig. 1) provide an insight into the mechanism responsible for the bonding between the $\mathrm{Eu}$ and $\mathrm{C}_{8} \mathrm{H}_{8}$ ligand molecules. In a qualitative picture, based on frontier orbital interactions arguments [19], when the $\mathrm{C}_{8} \mathrm{H}_{8}$ molecules and $\mathrm{Eu}$ atom are brought together, the highest occupied and lowest unoccupied $\pi$-type conjugated orbitals of $\mathrm{C}_{8} \mathrm{H}_{8}$ interact with the $\mathrm{Eu} 4 f$ orbitals of the same symmetry and hybridization occurs. Because of the strong aromatic character $[19,20]$ of the $\pi$ orbitals located at the $\mathrm{C}_{8} \mathrm{H}_{8}$ rings the $4 f$ electrons are drastically shifted in energy from the low part of the valence electrons towards the Fermi level [which is placed in the middle of the highest occupied molecular orbital-lowest unoccupied molecular orbital (HOMO-LUMO) gap]. This effect has been also observed in the experiments $[8,10]$. The new molecular orbitals, formed in a similar manner as previously shown for other sandwich-type systems (see [17] and the references therein), accommodate the $6 s$ as well as small fractions of $4 f$ electrons of the Eu atoms.

A picture of the electronic structure and the interaction of the Eu atoms due to the hybridization with the $\pi$ orbitals of the three rings is given in the following. In the first

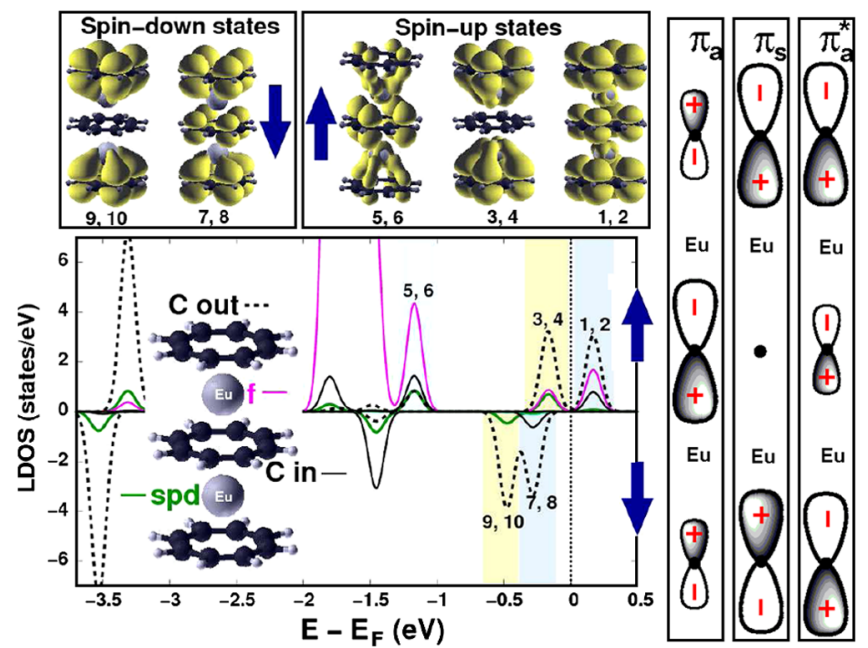

FIG. 1 (color online). Left panel: The ferromagnetic spinresolved density of states of the neutral $\mathrm{Eu}_{2}\left(\mathrm{C}_{8} \mathrm{H}_{8}\right)_{3}$ molecule $(U=3.7 \mathrm{eV})$ and the charge density plots of the states in the shaded areas. The $4 f$ electrons of Eu atom are drastically shifted from the core region towards Fermi level due to the hybridization with the aromatic orbitals of $\mathrm{C}_{8} \mathrm{H}_{8}$ rings. Right panel: Schematics of the symmetric $\left(\pi_{s}\right)$ and antisymmetric $\left(\pi_{a}, \pi_{a}^{*}\right)$ orbitals due to the hybridization of the $\pi$ orbitals of the three rings. The charge density plots $(5,6),(3,4)$, and $(1,2)$ correspond to $\pi_{a}, \pi_{s}$, and $\pi_{a}^{*}$ type orbitals, being twofold degenerate.

approximation the hybridization of the $\pi$ orbitals of the three different rings is neglected. The $\mathrm{Eu}^{2+}$ ions act as an attractive potential to the $\pi$ orbitals. Because of the two neighboring $\mathrm{Eu}^{2+}$ ions the $\pi$ orbitals of the central ring are strongly pulled down to lower energies and are fully occupied, providing two additional electrons to partially screen the two $\mathrm{Eu}^{2+}$ ions. These orbitals are about $1.5 \mathrm{eV}$ below the $\pi$ orbitals of the two outer rings, which experience only one attractive $\mathrm{Eu}^{2+}$ potential and provide together two more electrons in the neutral state of the $\mathrm{Eu}_{2}\left(\mathrm{C}_{8} \mathrm{H}_{8}\right)_{3}$; i.e., each outer ring has one additional electron. Thus the two $\mathrm{Eu}^{2+}$ ions are screened by the two electrons of the inner ring and one electron of each of the outer rings. For a more complete picture the hybridization of the $\pi$ orbitals of the three rings have to be included, as well as the hybridization with the $f$ orbitals. The HOMO and LUMO of the $\mathrm{C}_{8} \mathrm{H}_{8}^{-1}$ anion are the fourfold symmetric $\pi$ orbitals $[19,20]$ for which the rotational symmetry is preserved so that the model planes can be assumed to be common for all three rings (see Fig. 1, right panel). The $\pi$ orbitals of the rings can only hybridize with such $4 f$ states being compatible with the nodal plane symmetry $[17,19]$.

Because of symmetry, three types of orbitals arise as a result of the interaction between the $\mathrm{C}_{8} \mathrm{H}_{8}$ rings: symmetric $\left(\pi_{s}\right)$, bonding-antisymmetric $\left(\pi_{a}\right)$, and antibondingantisymmetric $\left(\pi_{a}^{*}\right)$. The $\pi_{s}$ states have a node at the inner ring, with most of the weight at the outer rings and a relatively large intensity at the Eu sites [the charge density plots 3, $4\left({ }^{\top} \pi_{s}\right)$ and $9,10\left({ }^{\downarrow} \pi_{s}\right)$ in Fig. 1]. The fully 
occupied $\pi_{a}$ orbitals appear at $1.0 \mathrm{eV}$ below $\pi_{s}$ orbitals and have larger amplitude at the inner ring [i.e., charge density plots $5,6\left({ }^{\dagger} \pi_{a}\right)$ in Fig. 1$]$. The ${ }^{\dagger} \pi_{a}^{*}$ orbitals (charge density plots 1,2 in Fig. 1) are localized mainly at the outer rings and are $0.36 \mathrm{eV}$ higher in energy than the ${ }^{\dagger} \pi_{s}$ orbitals, while the filled ${ }^{\downarrow} \pi_{a}^{*}$ orbitals appear at $-0.30 \mathrm{eV}$ below the Fermi level. The occupation of these orbitals around the Fermi energy as well as the resulting spin moment in the molecules as a function of the oxidation state from +2 up to -1 is presented in Fig. 2.

In the neutral compound, due to hybridization of the ${ }^{\dagger} \pi_{s}$ and ${ }^{\dagger} \pi_{a}^{*}$ orbitals with the $4 f$ orbitals, the ${ }^{\dagger} \pi_{s}$ orbitals are pushed to lower energies than the ${ }^{\dagger} \pi_{a}^{*}$ states which are pushed to higher energies and become empty. This leads to a net magnetic moment of $2 \mu_{B}$ carried by the $\pi$ orbitals. These orbitals are mostly localized on the two outer rings (for spin-down channel) and are antiparallel to the $\mathrm{Eu}$ magnetic moments of $7+7=14 \mu_{B}$, such that the total moment is $12 \mu_{B}$ in the $\mathrm{Eu}_{2}\left(\mathrm{C}_{8} \mathrm{H}_{8}\right)_{3}$. The redistribution of the empty and occupied molecular orbitals around the Fermi level, which is a consequence of the hybridization with the transition metal ions, is due to the $\pi-f$ Zener type exchange mechanism [21], which is responsible for the ferromagnetism in dilute magnetic semiconductors (DMS) like $(\mathrm{Ga}, \mathrm{Mn}) \mathrm{As}$ or $(\mathrm{In}, \mathrm{Mn}) \mathrm{Sb}$ [22]. As in DMS systems, the ferromagnetism in the $\mathrm{Eu}_{2}\left(\mathrm{C}_{8} \mathrm{H}_{8}\right)_{3}$ molecule is hole mediated and caused by the $\pi-f$ exchange in analogy to $p-d$ exchange in $(\mathrm{Ga}, \mathrm{Mn}) \mathrm{As}$. In the $\mathrm{Eu}_{2}\left(\mathrm{C}_{8} \mathrm{H}_{8}\right)_{3}^{-2}$ molecule all $\pi_{a}, \pi_{s}, \pi_{a}^{*}$ orbitals for both spin channels become occupied and the redistribution of the molecular states due to interaction with Eu ions does not bring any gain in total energy as compared to the antiferromagnetic spin alignment.

Characteristic for all ionic species when the magnetization points in-plane $(r)$ the 1,2 orbitals $\left({ }^{\uparrow} \pi_{a}^{*}\right)$ have mainly $f_{m_{l}=-2}$ orbital character while $3,4\left({ }^{\dagger} \pi_{s}\right)$ orbitals have mainly $f_{m_{l}=+1}, f_{m_{l}=+3}$ contributions. The spin-orbit splitting is small and leaves the value of the HOMO-LUMO gaps unaffected. Orienting the magnetization in the $z$ direction results in a lifting of the symmetry allowing the $f_{m_{l}=-2}, f_{m_{l}=+1}$, and $f_{m_{l}=+3}$ states on two Eu atoms to hybridize. The effect of the spin-orbit coupling is quite large, leading to an effective splitting between the states 1 , $2\left({ }^{\dagger} \pi_{a}^{*}\right)$ and $3,4\left({ }^{\dagger} \pi_{s}\right)$ and a significant change of the corresponding HOMO-LUMO gaps.

We would like to point out that the stabilization of a specific magnetization direction is a consequence of the electronic structure at the Fermi level: as compared to other magnetization directions a higher orbital moment $\mu_{L}$ is the result of a larger spin-orbit splitting of the energy levels (see Fig. 3) due to a stronger bonding in the molecule. This situation is analogous to that in the infinite molecular $\left(\mathrm{Nb}-\mathrm{C}_{6} \mathrm{H}_{6}\right)_{\infty}$ wire, for which orienting the magnetization in the plane of benzenes lowers the energy of one of the spin-orbit-split bands [17] and results in the largest value of $\mu_{L}$. The same correlation we observe in our $\mathrm{Eu}_{2}\left(\mathrm{C}_{8} \mathrm{H}_{8}\right)_{3}$ molecule and its ionic species: for the $\mathrm{Eu}_{2}\left(\mathrm{C}_{8} \mathrm{H}_{8}\right)_{3}$ and $\mathrm{Eu}_{2}\left(\mathrm{C}_{8} \mathrm{H}_{8}\right)_{3}^{+2}$ the HOMO-LUMO gap and $\mu_{L}$ are larger for the $r$ magnetization (the anisotropy energy favors the in-plane magnetization by a value of 2.68 and $2.58 \mathrm{meV}$, respectively), while for $\mathrm{Eu}_{2}\left(\mathrm{C}_{8} \mathrm{H}_{8}\right)_{3}^{+1}$ and $\mathrm{Eu}_{2}\left(\mathrm{C}_{8} \mathrm{H}_{8}\right)_{3}^{-1}$ the HOMO-LUMO gap and $\mu_{L}$ are larger when the magnetization points in $z$ direction (the anisotropy energy favors the perpendicular to plane magnetization by a value of 1.91 and $13.67 \mathrm{meV}$, respectively) [23]. The larger orbital moment of the $\mathrm{Eu}_{2}\left(\mathrm{C}_{8} \mathrm{H}_{8}\right)_{3}^{-1}$ anion as compared to the other ionic species arises from the stronger hybridization of the $\pi_{a}^{*}$ orbitals with the $f$ states and results in a strong increase in the anisotropy value according to the expression for MAE in the perturbation theory [24].

On the contrary, the double negatively charged $\mathrm{Eu}_{2}\left(\mathrm{C}_{8} \mathrm{H}_{8}\right)_{3}^{-2}$ has both $1,2\left({ }^{\dagger} \pi_{a}^{*}\right)$ levels filled and conse-

\begin{tabular}{|c|c|c|c|c|c|c|c|}
\hline \multicolumn{2}{|c|}{$\begin{array}{c}\text { Cation } \\
\mathrm{Eu}_{2}\left(\mathrm{C}_{8} \mathrm{H}_{8}\right)_{3}^{+2}\end{array}$} & \multicolumn{2}{|c|}{$\begin{array}{l}\text { Cation } \\
\mathrm{Eu}_{2}\left(\mathrm{C}_{8} \mathrm{H}_{8}\right)_{3}{ }^{+1}\end{array}$} & \multicolumn{2}{|c|}{$\begin{array}{c}\text { Neutral } \\
\mathrm{Eu}_{2}\left(\mathrm{C}_{8} \mathrm{H}_{8}\right)_{3}\end{array}$} & \multicolumn{2}{|c|}{$\begin{array}{c}\text { Anion } \\
\mathrm{Eu}_{2}\left(\mathrm{C}_{8} \mathrm{H}_{8}\right)_{3}^{-1}\end{array}$} \\
\hline spin down & $\frac{\text { spin up }}{\frac{1}{\frac{1}{3}} \frac{2}{4} \pi_{a}^{*}}$ & spin down & $\frac{\text { spin up }}{\frac{1}{3}^{2} \pi_{a}^{*} \pi_{s}}$ & \begin{tabular}{|l} 
spin down \\
\end{tabular} & $\frac{\text { spin up }}{1 \frac{\pi}{2} \pi_{a}^{*}}$ & \begin{tabular}{|l} 
spin \\
\end{tabular} & $\frac{\text { spin up }}{\frac{1}{1} \pi_{a}^{*}}$ \\
\hline $\begin{array}{l}\pi_{a}^{* 78} 8 \\
\pi_{s} 910\end{array}$ & & $\begin{array}{l}\pi_{a}^{* 7} 8 \\
\pi_{s}=10\end{array}$ & $\frac{1}{4} \pi_{s}$ & $\begin{array}{l}\pi_{a 7}^{* 7} 8 \\
\pi_{s}^{9} 10\end{array}$ & $\frac{1}{3} \frac{1}{4} \pi_{s}$ & $\begin{array}{l}\pi_{a}^{* 7} 8 \\
\pi_{s} \mathbf{9} 10\end{array}$ & $\frac{1}{3} \frac{1}{4} \pi_{s}$ \\
\hline $7 \hat{+}+7$ & $=10$ & $7 \hat{+}+$ & $\begin{array}{l}\text { spin } m \\
3=11\end{array}$ & $\begin{array}{l}(\mu \mathrm{B}) \\
7 \hat{\dagger}+7 \hat{1}\end{array}$ & $=12$ & $7 \hat{+}+7 \hat{\imath}$ & $1=13$ \\
\hline $\begin{array}{l}\text { MAE } \\
(\mathrm{meV})\end{array}$ & lane & ext & $\begin{array}{l}1.91 \\
\text { pendicular }\end{array}$ & et: & plane & 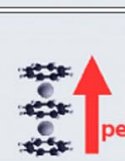 & $\begin{array}{l}13.67 \\
\text { pendicular }\end{array}$ \\
\hline 0.0 & & & $\begin{array}{l}\mu_{L}=\mid \mu_{L}(r \\
68\end{array}$ & $\begin{array}{r}\mu_{L}(z) \mid(\mu \mathrm{B} \\
0\end{array}$ & 34 & & \\
\hline
\end{tabular}

FIG. 2 (color online). Schematic view of the highest occupied and the lowest unoccupied energy levels of $\mathrm{Eu}_{2}\left(\mathrm{C}_{8} \mathrm{H}_{8}\right)_{3}$ ionic species. The anisotropy energy (MAE) favors the in-plane magnetization $(r)$ for the $\mathrm{Eu}_{2}\left(\mathrm{C}_{8} \mathrm{H}_{8}\right)_{3}$ and $\mathrm{Eu}_{2}\left(\mathrm{C}_{8} \mathrm{H}_{8}\right)_{3}^{+2}$, while the perpendicular to plane magnetization $(z)$ is favored for the $\mathrm{Eu}_{2}\left(\mathrm{C}_{8} \mathrm{H}_{8}\right)_{3}^{+1}$ and $\mathrm{Eu}_{2}\left(\mathrm{C}_{8} \mathrm{H}_{8}\right)_{3}^{-1}$. The orbital moment $\mu_{L}$ is always larger for the favored magnetization direction by a value $\Delta \mu_{L}$. 




FIG. 3 (color online). The highest occupied and the lowest unoccupied molecular orbitals of $\mathrm{Eu}_{2}\left(\mathrm{C}_{8} \mathrm{H}_{8}\right)_{3}$ and $\mathrm{Eu}_{2}\left(\mathrm{C}_{8} \mathrm{H}_{8}\right)_{3}^{-1}$ without and with spin-orbit coupling (SOC) for two magnetization directions are presented. The energy levels for $\mathrm{Eu}_{2}\left(\mathrm{C}_{8} \mathrm{H}_{8}\right)_{3}$ and $\mathrm{Eu}_{2}\left(\mathrm{C}_{8} \mathrm{H}_{8}\right)_{3}^{-1}$ are shown as follows: left panel-without SOC; middle panel-SOC in $r$ direction; right panel-SOC in $z$ direction. The larger spin-orbit splitting value of the HOMOLUMO gap corresponds to a stronger bonding in the molecule and depends on the magnetization direction, as well as oxidation state. The orbitals 2 and 4 are obtained by rotating orbitals 1 and 3 , respectively, around the $z$ axis by $\pi / 4$.

quently the $\mu_{L}$ and MAE values become negligible. Moreover, our calculations on the infinite wire $\mathrm{Eu}\left(\mathrm{C}_{8} \mathrm{H}_{8}\right)_{\infty}$ show that the energy difference between ferromagnetic and antiferromagnetic state is $2.5 \mathrm{meV}$ per $\mathrm{Eu}$ atom and the value of the MAE is of the order of $10^{-3} \mathrm{meV}$. Thus, in the $\mathrm{Eu}_{2}\left(\mathrm{C}_{8} \mathrm{H}_{8}\right)_{3}$ molecule and its ionic species the $\pi$ orbitals of the outer rings are responsible for the large MAE values and change of the magnetization direction. We emphasize that these changes are caused by the finite dimensions of the molecules (surface effect).

To conclude, the "raptor" nature of the $\mathrm{C}_{8} \mathrm{H}_{8}$ ligand almost removes the Eu $6 s$ electrons from their nucleus and place them in $\pi$-type orbitals. The $4 f$ electrons are dragged closer to the Fermi energy by hybridizing with the $\pi$-type orbitals of the $\mathrm{C}_{8} \mathrm{H}_{8}$ rings. The new molecular orbitals which are formed have a stronger aromatic character leading to a stronger bonding and greater stabilization of the entire system. Therefore, the $4 f$ electrons of the two $\mathrm{Eu}$ atoms are able to contribute slightly to the bonding and couple ferromagnetically via a Zener's $\pi-f$ exchange. The phenomenal effect predicted by our calculations is the change of the magnetization direction from in-plane $(r)$ to axial $(z)$ by charging or decharging the $\mathrm{Eu}_{2}\left(\mathrm{C}_{8} \mathrm{H}_{8}\right)_{3}$ molecule. This novel effect together with the ferromagnetic coupling could be used in many technological applications, for instance in data storage [1] or in the single electron transistor using a single redox active organic molecule [3].
The computations were performed with the help of the JUMP and Blue/Gene supercomputers at the Forschungszentrum Jülich, Germany. We acknowledge financial support from the DFG Grant No. SPP1243.

*n.atodiresei@fz-juelich.de

[1] S. Maekawa and T. Shinjo, Spin Dependent Transport in Magnetic Structures (Taylor \& Francis Group, London, 2002).

[2] A. R. Rocha, V. M. Garcia-Suarez, S. Bailey, C. Lambert, J. Ferrer, and S. Sanvito, Nat. Mater. 4, 335 (2005).

[3] S. Kubatkin, A. Danilov, M. Hjoer, J. Cornil, J. L. Bredas, N. Sthur-Hansen, and T. B. P. Hedegard, Nature (London) 425, 698 (2003).

[4] M. A. Kastner, Rev. Mod. Phys. 64, 849 (1992).

[5] C. Chappert, A. Fert, and F. N. van Dau, Nat. Mater. 6, 813 (2007).

[6] A. Moser, K. Takano, D. T. Margulies, M. Albrecht, Y. Sonobe, Y. Ikeda, S. Sun, and E. E. Fullerton, J. Phys. D 35, R157 (2002).

[7] C. Thirion, W. Wernsdorfer, and D. Mailly, Nat. Mater. 2, 524 (2003).

[8] N. Hosoya, R. Takegami, J. Suzumura, K. Yada, K. Koyasu, K. Miyajima, M. Mitsui, M. B. Knickelbein, S. Yabushita, and A. Nakajima, J. Phys. Chem. A 109, 9 (2005).

[9] K. Miyajima, T. Kurikawa, M. Hashimoto, A. Nakajima, and K. Kaya, Chem. Phys. Lett. 306, 256 (1999).

[10] R. Takegami, N. Hosoya, J. Suzumura, A. Nakajima, and S. Yabishita, J. Phys. Chem. A 109, 2476 (2005).

[11] W. Kohn and L. J. Sham, Phys. Rev. 140, A1133 (1965).

[12] J. P. Perdew, K. Burke, and M. Ernzerhof, Phys. Rev. Lett. 77, 3865 (1996).

[13] P. E. Blöchl, Phys. Rev. B 50, 17953 (1994).

[14] G. Kresse and J. Furthmüller, Comput. Mater. Sci. 6, 15 (1996).

[15] V. I. Anisimov, F. Aryasetiawan, and A. I. Lichtenstein, J. Phys. Condens. Matter 9, 767 (1997).

[16] P. Kurz, G. Bihlmayer, and S. Blügel, J. Phys. Condens. Matter 14, 6353 (2002).

[17] Y. Mokrousov, N. Atodiresei, G. Bihlmayer, and S. Blügel, Int. J. Quantum Chem. 106, 3208 (2006).

[18] W. T. B.P. G. Wenthold and D. A. Hrovat, Science 272, 1456 (1996).

[19] A. Rauk, Orbital Interaction Theory of Organic Chemistry (John Wiley \& Sons, New York, 2001).

[20] J. H. Hammons, D. A. Hrovat, and W. T. Borden, J. Am. Chem. Soc. 113, 4500 (1991).

[21] T. Dielt, Semicond. Sci. Technol. 17, 377 (2002).

[22] J. Kanamori and K. Terakura, J. Phys. Soc. Jpn. 70, 1433 (2001).

[23] The effect of $U$ can be seen as follows: by increasing the $U$, the $f$ states become more localized and atomiclike. The hybridization is reduced, the value of the orbital moment vanishes and the HOMO-LUMO gap becomes irrespective to the magnetization direction. All these lead to the overall reduction in the values of MAE.

[24] P. Bruno, Phys. Rev. B 39, 865 (1989). 